\title{
Quercetin and epigallocatechin gallate effects on the cell membranes biophysical properties correlate with their antioxidant potential
}

\author{
Denisa Margina $^{1}$, Mihaela Iliee ${ }^{1}$, Gina Manda², Ionela Neagoe ${ }^{2}$, Magdalena Mocanu ${ }^{3}$, Diana \\ Ionescu $^{3}$, Daniela Gradinaru ${ }^{1}$ and Constanța Ganea ${ }^{3}$ \\ ${ }^{1}$ Carol Davila University of Medicine and Pharmacy, Faculty of Pharmacy, 6 Traian Vuia, sect 2, Bucharest, Romania \\ ${ }^{2}$ Victor Babeş National Institute for Pathology and Biomedical Sciences, Bucharest, 99-101 Splaiul Independenței, 050096 \\ Bucharest, Romania \\ ${ }^{3}$ Carol Davila University of Medicine and Pharmacy, Faculty of Medicine, 8 Eroii Sanitari Blvd., sect 5, Bucharest, Romania
}

\begin{abstract}
Quercetin and epigallocatechin gallate are two of the most abundant polyphenols in dietary plants, including apples, onions, red wine and green tea. The bioactivity of polyphenols is linked to their ability to interact with cell membranes without being internalized. The aim of the present study was to assess the short-time effect of these polyphenols on membrane anisotropy and transmembrane potential of U937 monocytes and Jurkat T lymphoblasts. Results showed that quercetin and epigallocatechin gallate induced, after 20 minutes cell exposure, a dose-dependent increase of membrane anisotropy and polarization. Anisotropy increase was correlated with the reduction of lipid peroxidation. Our results could indicate that the antioxidant capacity of the tested polyphenols is due to their stabilizing effect on the cell membranes, thus contributing to cell protection in various pathologies and as adjuvant therapy in highly toxic treatment regimens.
\end{abstract}

Key words: Polyphenols - Membrane potential - Membrane anisotropy — Antioxidant potential

\begin{abstract}
Abbreviations: $\mathrm{DiBAC}_{4}(3)$, bis-(1,3 -dibutylbarbituric acid)trimethine oxonol; DMSO, dimethyl sulfoxide; DPPP, diphenyl-1-pyrenylphosphine; EGCG, epigallocatechin gallate; FCS, fetal calf serum; PBS, phosphate buffer saline; TMA-DPH, 1-(4-trimethylammoniumphenyl)-6-phenyl-1,3,5hexatriene p-toluensulfonate.
\end{abstract}

\section{Introduction}

The health-promoting effects of vegetable-based diets have been attributed to their rich polyphenol composition (Boots et al. 2008; Fraga et al. 2010). Quercetin and epigallocatechin gallate (EGCG) are two of the most abundant polyphenol compounds found in foods as apples, onions, red wine and green tea. According to literature data, quercetin and EGCG can modulate intracellular calcium concentration, thus preventing cell oxidative processes and other $\mathrm{Ca}^{2+}$-regulated events as well (Verstraeten et al. 2008; Sánchez-Gallego et al. 2010). Last years research proved the involvement of flavo-

Correspondence to: Mihaela Ilie, Carol Davila University of Medicine and Pharmacy, Faculty of Pharmacy, 6 Traian Vuia, sect 2, Bucharest, Romania

E-mail: m16ilie@yahoo.com noids in modulating oxidative stress and other pathological processes, including inflammation. These studies could explain the reason for their beneficial effects for human health such as prevention of atherosclerosis-based cardiovascular disorders, diabetes and other metabolic diseases or even cancer (Arts et al. 2002; Taubert et al. 2003; Manach et al. 2004; Vita 2005; Schroeter et al. 2006; Mursu et al. 2008).

The bioactivity of flavonoids is closely linked to their ability to interact with membranes since many of these compounds generate a cell response even if they are not internalized (Erlejman et al. 2008; Verstraeten et al. 2008). For example, the location of the polyphenolic structure at the membrane level may result in changes that allow them to sterically hinder the diffusion of free radicals, therefore the interaction with cell membranes might be a decisive factor for their antioxidant activity (Arora et al. 2000; Barros et al. 2001; Oteiza et al. 2005; Sánchez-Gallego et al. 2010). 
Membrane microviscosity and transmembrane potential are among the most important biophysical properties of cell membranes. There are several research reports that link the microviscosity of cell membranes to the effects induced by different toxic stimuli. The increase of hepatocytes membrane fluidity under the influence of some xenobiotics (such as tacrine, ximelagatran or ethanol) is related to drug-induced liver injury and is leading to substantial disturbances of cell function (Sergent et al. 2005, 2009). Fujimoto et al. (1999) proved that cells exposed to apoptotic stimuli exhibit alterations of membrane fluidity. The viral infection of N2a mouse neuroblastoma cells leads to a significant increase in membrane fluidity (Mishra et al. 2009). Incubation with oxidized LDL particles induced an increase of the endothelial cells membrane fluidity and a-tocopherol prevented this effect (Ghosh et al. 2002; van Aalst et al. 2004).

Several studies investigated the interaction between the two polyphenols and the cell membrane using both artificial membranes (Kajiya et al. 2002; Kumazawa et al. 2004; Tamba et al. 2007; Kamihara et al. 2008; Iftime et al. 2010) and cell lines (Kanupriya et al. 2006; Verstraeten et al. 2008) and the results showed that flavonoids induced changes at the cell membrane level that lead to an increased protection against oxidative stress.

The aim of the present study was to assess early effects exerted in vitro by quercetin and EGCG on membrane anisotropy and transmembrane potential of Jurkat and U937 cell lines, correlated with the antioxidant activity of the two polyphenols.

Our results suggest that the antioxidant capacity of the tested polyphenols is due to their stabilizing effect on the cell membranes, thus contributing to cell protection in various pathologies and as adjuvant therapy in highly toxic treatment regimens. These studies could have an impact on the global tendency of using polyphenols in preventive and curative medicine.

\section{Materials and Methods}

\section{Materials}

HPLC grade EGCG (purity $\geq 95 \%$, from Sigma, code E4143) and quercetin (from Merck) were used for the in vitro stimulation of the cells; in the case of EGCG, the content of up to $5 \%$ unknown polyphenolic substances can influence the results. The stock solutions $(10 \mathrm{mM}$ quercetin in DMSO and EGCG in water) were kept at $-20^{\circ} \mathrm{C}$ between different day experiments. RPMI 1640 (supplemented with L-gluthamate) from Merck was used as the main medium for the cells suspension during all tests performed.

1-(4-trimethylammoniumphenyl)-6-phenyl-1,3,5-hexatriene $\mathrm{p}$-toluensulfonate (TMA-DPH) was purchased from
Molecular Probes (Invitrogen Inc.) and used as a membrane anisotropy fluorescence probe. Bis-(1,3-dibutylbarbituric acid)trimethine oxonol $\left(\mathrm{DiBAC}_{4}(3)\right)$, was bought from Molecular Probes and was used as a fluorescent probe for the evaluation of transmembrane potential changes under the influence of the two polyphenols. Diphenyl-1-pyrenylphosphine (DPPP) from Molecular Probes was used as a fluorescent probe to monitor cumene hydroperoxide $(\mathrm{CuOOH}) 10 \mu \mathrm{M}$ (Sigma)-induced lipid peroxidation of the cell membranes.

The cell membrane anisotropy and transmembrane cell potential evaluation were performed using a LS 50B Perkin Elmer spectrofluorimeter equipped with thermostated cell holder, magnetic stirring and fluorescence polarization accessory in a double-staining experiment. The lipid peroxidation was monitored in dynamics, using the DPPP bound to cells fluorescence spectra recorded on the Perkin Elmer spectrofluorimeter (model LS 50B) as well.

\section{Cell cultures}

Experiments were performed using U937 and Jurkat cells, from European Collection of Cell Cultures, at the 3th-4th passage. The U937 human macrophage cell line as well as Jurkat lymphocytic cells were grown in RPMI 1640 supplemented with $10 \%$ heat-inactivated fetal calf serum (FCS) at $37^{\circ} \mathrm{C}$ in $5 \% \mathrm{CO}_{2}$ atmosphere.

Prior to the experiments, cells were washed 3 times with phosphate buffer saline (PBS; pH 7) at $1200 \mathrm{rpm}$, for 2 minutes. After the last centrifugation, they were counted and standardized at $10^{5}$ cells $/ \mathrm{ml}$ to be used in aliquots of $2 \mathrm{ml}$ in the fluorimeter cuvette. Results are the mean of three replicate determinations.

\section{Cell membrane fluidity assay}

The cell membrane anisotropy measurements were performed by evaluation of the anisotropy value of TMA-DPH in a steady state fluorescence polarization experiment. TMA$\mathrm{DPH}$ is a classically used probe to assess the membrane-like local anisotropy of a fluidic microsystem (Van Blitterswijk et al. 1981; Huang and Haugland 1991; Pebay-Peyroula et al. 1994; Martins et al. 2010). We determined the TMA$\mathrm{DPH}$ bound to cell anisotropy, as fluidity is proportional to the inverse of squared anisotropy (Pebay-Peyroula et al. 1994) and the results were expressed as ratios with respect to a control.

The fluorescence anisotropy of labeled cells was evaluated prior and after 20 minutes of incubation, at room temperature, with quercetin or EGCG. For this purpose, the normalized cell suspension labeled with $2 \mu \mathrm{M}$ TMA-DPH ( 2 minutes incubation at $37^{\circ} \mathrm{C}$, under continuous magnetic stirring) was excited with polarized light at $340 \mathrm{~nm}$ and the 
emission intensities were detected at $425 \mathrm{~nm}$. The measurements were carried out for the four possible positions of the polarizers in the excitation and emission beam. A control sample consisting in non-treated cells was identically evaluated (Ilie et al. 2009).

The calculation of the fluorescence anisotropy $(r)$ was performed according to equation:

$$
r=\frac{I_{v v}-G \cdot I_{v h}}{I_{v v}+2 \cdot G \cdot I_{v h}}
$$

where $G=I_{h v} / I_{h h}$ is an instrumental factor, and $I_{v v}, I_{v h}$, $I_{h v}$ and $I_{h h}$ represent the emission intensity when the polarizers are oriented vertical-vertical, vertical-horizontal, horizontal-vertical and horizontal-horizontal, respectively (Lakowicz 1999).

The effect was evaluated as relative anisotropy change effect following equation:

$$
e_{r}(\%)=100 \cdot\left(\frac{r_{i}}{r_{c}}-1\right)
$$

where $r_{i}$ is the anisotropy value of the sample and $r_{c}$ is the anisotropy value of the control sample.

\section{Transmembrane potential evaluation}

$\mathrm{DiBAC}_{4}(3)$ is a slow-response potential-sensitive probe that can enter depolarized cells, binds to intracellular proteins or membrane thus exhibiting enhanced fluorescence (Epps et al. 1994). An increase in depolarization generates an additional influx of the anionic dye, resulting in the increase of fluorescence. Conversely, hyperpolarization (increase in the absolute value of the membrane potential) is indicated by a decrease in fluorescence (Klapperstück et al. 2009).

In a normalized suspension $(2 \mu \mathrm{M}$ probe concentration for $10^{5}$ cells $\left./ \mathrm{ml}\right), \operatorname{DiBAC}_{4}(3)$-labeled cells were excited at $493 \mathrm{~nm}$ and fluorescence emission spectra were collected within the range 500 to $600 \mathrm{~nm}$. Fluorescence intensity was measured at $517 \mathrm{~nm}$ before and after 20 minutes incubation of the cells with quercetin or EGCG. A control sample of untreated cells was also evaluated similar to the samples. The membrane potential changes were evaluated as a relative effect of the two polyphenols using equation:

$$
e_{v}(\%)=100 \cdot\left(1-\frac{I_{i}-I_{o i}}{I_{c}-I_{o c}}\right)
$$

where I represent the fluorescence emission intensity measured at $517 \mathrm{~nm}$, the indices $i$ and $c$ denotes the treated sample and the control, respectively, and the index 0 denotes the signal before the incubation with the dye.
In order to reduce the number of experiments, the two fluorescent probes were added simultaneously, and the fluorescent signals were collected successively, after performing tests that showed that the two probes do not interfere, either spectrally or chemically (results not shown) and the final results were not statistically interfered by the successive measurements of the signals.

\section{Measurement of membrane hydroperoxides by DPPP-oxide fluorescence}

Several methods for the evaluation of lipid peroxidation are mentioned in literature data (Breusing et al. 2010; Margina et al. 2011). DPPP is a probe that lacks fluorescence; its oxide, which is specifically generated in reaction with hydroperoxides, has strong fluorescence (Gomes et al. 2005). According to literature data, experimental lipid peroxidation was induced by $10 \mu \mathrm{M} \mathrm{CuOOH}$ which generates an effective reaction with DPPP in the membrane (Takahashi et al. 2001).

DPPP was dissolved in DMSO at a concentration of $5 \mathrm{mM}$. After addition of DPPP at a final concentration of $5 \mu \mathrm{M}$, cell suspension was incubated at room temperature, for 20 minutes in the dark. DPPP-labeled cells were treated with $10 \mu \mathrm{M} \mathrm{CuOOH}$ to induce DPPP-oxide formation. The process was assayed by performing fluorescence emission spectra between $360 \mathrm{~nm}$ and $410 \mathrm{~nm}$, excitation at $351 \mathrm{~nm}$, every minute, for 5 minutes. Fluorescence intensity maximum was measured at $380 \mathrm{~nm}$ (Okimoto et al. 2000) and represented as a time function.

\section{Statistical analysis}

Results are expressed as means \pm standard deviation (SD). Statistical analysis was performed using the Student's $t$-test. Differences were considered significant for $p<0.05$.

\section{Results}

The aim of the present study was to assess short-time effects exerted in vitro by quercetin and EGCG on membrane anisotropy and transmembrane potential of Jurkat and U937 cell lines, correlated with the antioxidant activity of the two polyphenols.

We selected human constitutively proliferating cell lines (Jurkat T lymphoblasts and U937 monocytes) corresponding to peripheral leukocytes, in order to generate knowledge regarding the effect of quercetin and EGCG by in vitro studies on cell lines to be further translated to in vivo human studies.

The cellular parameters investigated in this study were chosen considering that cell functions are highly dependent 
on membrane integrity, involving proper anisotropy, transmembrane potential and low levels of lipid peroxides.

\section{Setup of the experimental model}

Preliminary experiments showed that tested compounds (quercetin and EGCG) did not interfere with the fluorescent probes as they have different maximum emission wavelength (not shown). Personal unpublished data demonstrated that the fluorescent signals of the dyes used for anisotropy (TMA$\mathrm{DPH})$ and transmembrane potential $\left(\mathrm{DiBAC}_{4}(3)\right)$ did not interfere with each other.

For setting up the in vitro protocol, cells were incubated for 5, 10, 20 and 30 minutes with the tested compounds and we observed that the maximum effect was registered at 20 minutes; the anisotropy and transmembrane potential did not change at longer incubation time.

As such, our experimental model is appropriate for testing in vitro the two selected polyphenols.

\section{Effects of quercetin and EGCG on U937 and Jurkat mem- brane anisotropy}

TMA-DPH was designed to allow a more precise positioning of the probe at the membrane level compared to its earlier congener - DPH, since the cationic trimethylammonium group acts as a surface anchor. The probe displays, in isotropic media, specific fluorescence anisotropy properties, which are changed if the free orientation of the probe is restricted by the local microenvironment. This is the basis on which the probe functions to assess the changes in the

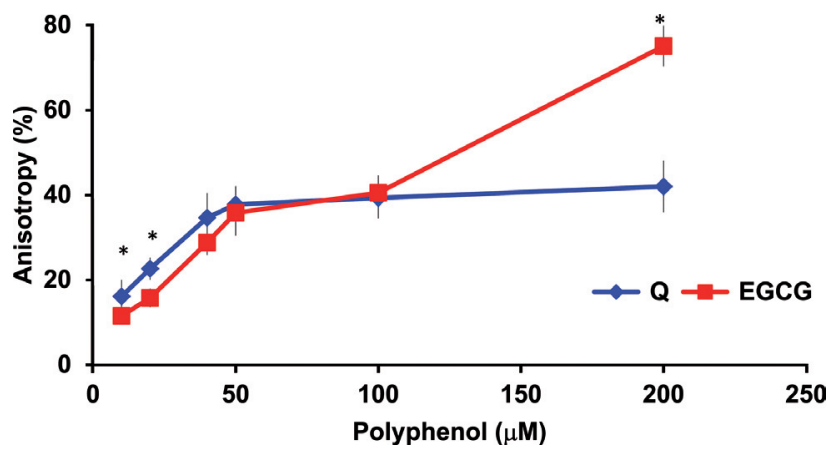

Figure 1. Quercetin (Q) and EGG effects on the membrane anisotropy of U937 cells, expressed as \% increase reported to the unstimulated cells. Q and EGCG induce a significant increase of the U937 membrane anisotropy, at concentrations up to $50 \mu \mathrm{M}$; at higher levels of exposure, we have observed a limitation of the effect in the case of Q. EGCG induces a $40 \%$ increase of the anisotropy, when rising from $100 \mu \mathrm{M}$ to $200 \mu \mathrm{M}$. Results are expressed as \% increase reported to the unstimulated cells; significantly statistic results are marked with an asterisk. microviscosity of the cell membrane: after the cell membrane exterior phospholipid layer permeation, the probe binds to the membrane and becomes a "rapporteur" for the changes in the anisotropy of the membrane.

The short-time exposure of cells to different concentrations of each of the two polyphenols used lead to an increase of the membrane anisotropy (Figure 1). As anisotropy and fluidity are inversely correlated, we can say that quercetin and EGCG induced a dose-dependent decrease of the U937 membrane fluidity; the EGCG effect was stronger than the effect exerted byquercetin, for the highest level of the tested concentrations.

In order to see if the results are the same on other nonadherent cell line, we evaluated the dose-dependent effects of quercetin and EGCG on the membrane of Jurkat cells (Figure 2). In the case of Jurkat cells, results show that the two polyphenols induced the increase of the anisotropy, as well. In order to exclude the effect induced by DMSO (as quercetin was dissolved in this solvent), the effect induced by $20 \mu \mathrm{l}$ of solvent on the membrane anisotropy was tested for both types of cells. This solvent volume was equivalent to the amount of DMSO used for the $100 \mu \mathrm{M}$ of quercetin solution added to the cells. The solvent induced a $0.28-0.30 \%$ effect, which can be considered negligible; therefore the increase in the cell membrane rigidity (decrease in fluidity) was entirely attributed to quercetin (dissolved in DMSO).

Effect of quercetin and EGCG on the transmembrane potential of U937 and Jurkat cells

The tested compounds induced the reduction of the fluorescence emission intensity of $\mathrm{DiBAC}_{4}(3)$ after membrane permeation, so they hyperpolarized the membranes of U937

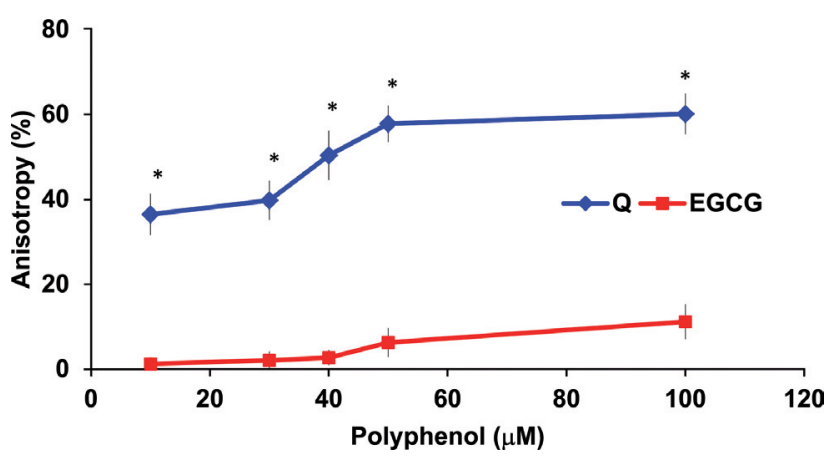

Figure 2. Quercetin (Q) and EGCG effects on membrane anisotropy of Jurkat cells, expressed as \% increase reported to the unstimulated cells. Q induces a $36.45-57.79 \%$ increase in the anisotropy value, when used in concentrations up to $50 \mu \mathrm{M}$; at higher levels of exposure, the anisotropy increase is very small. The effect of EGCG is significantly lower $(11.16 \%$ increase at 100 $\mu \mathrm{M})$; significantly statistic results are marked with an asterisk. 


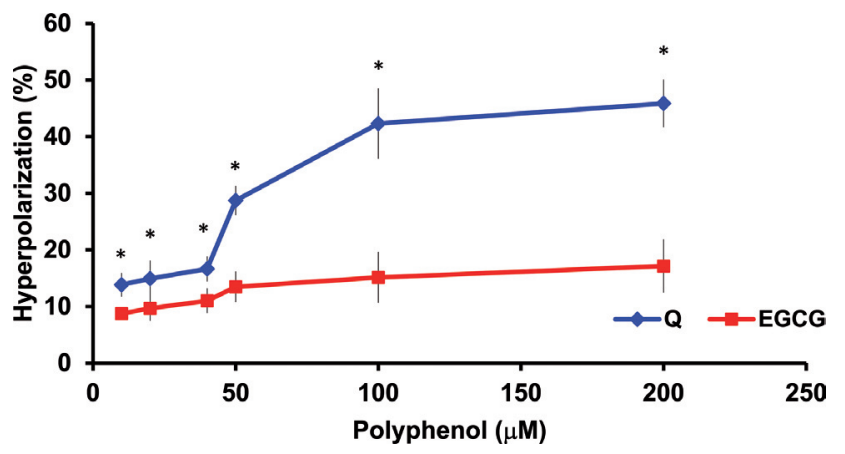

Figure 3. Hyperpolarizing effects induced by quercetin (Q) and EGCG on U937 cell membrane, compared to the unstimulated cells. Up to about $50 \mu \mathrm{M}$, the two polyphenols induce a gradual hyperpolarizing effect. At higher levels of exposure, Q induces a very important hyperpolarization (17.14\%), while EGCG resulted in only a gradual increase of transmembrane potential (3.67\%); significantly statistic results are marked with an asterisk.

cells. The effects of both compounds were dose-dependent. The quercetin effect was much stronger than that induced by EGCG (Figure 3).

Experiments performed on the Jurkat cell line proved the same hyperpolarizing effect exerted by the polyphenols. The effect induced by EGCG in this case was stronger than the one induced by quercetin, but the aspect of the hyperpolarizing curves was similar for the two cell lines (Figure 4).

Evaluation of the Jurkat membrane lipid peroxidation using $D P P P$

For the Jurkat cell line, we tested the effect of the polyphenol incubation on the peroxidation of the lipid membranes induced with $\mathrm{CuOOH}$. Incubation of the cells for 20 minutes with quercetin and EGCG reduced the level of DPPP-peroxide

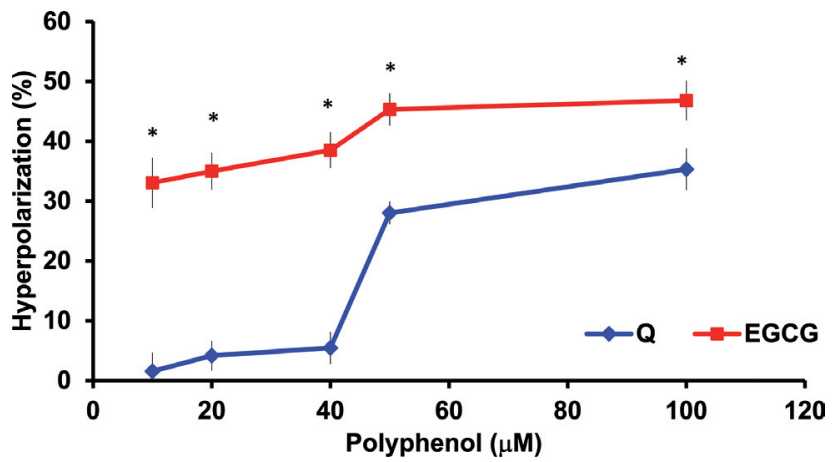

Figure 4. Hyperpolarizing effects induced by quercetin $(\mathrm{Q})$ and EGCG on Jurkat cell membrane, compared to the unstimulated cells. Concentration profiles of hyperpolarization of Jurkat cells for both polyphenols are similar to those observed in U937 cells, with the difference that the level of hyperpolarization is markedly different at low concentrations of polyphenols. EGCG is significantly more efficient that Q; significantly statistic results are marked with an asterisk.

generation (Figure 5). The increase of the stimuli concentration, from $10 \mu \mathrm{M}$ to $100 \mu \mathrm{M}$, reduced significantly the DPPP fluorescent signal, permitting us to believe that DPPP-peroxide generation was inhibited. In order to evaluate whether, in the case of quercetin, the antioxidant effect is due to the polyphenol itself and not to its solvent (DMSO), we also prepared a control sample where the cells were treated with the same amount of solvent added with the highest tested quercetin concentration of $100 \mu \mathrm{M}$. Results show that DMSO did not change the redox status in the cell suspension.

\section{Discussions}

An understanding of the effects of flavonoids and isoflavonoids on biophysical properties of membranes may

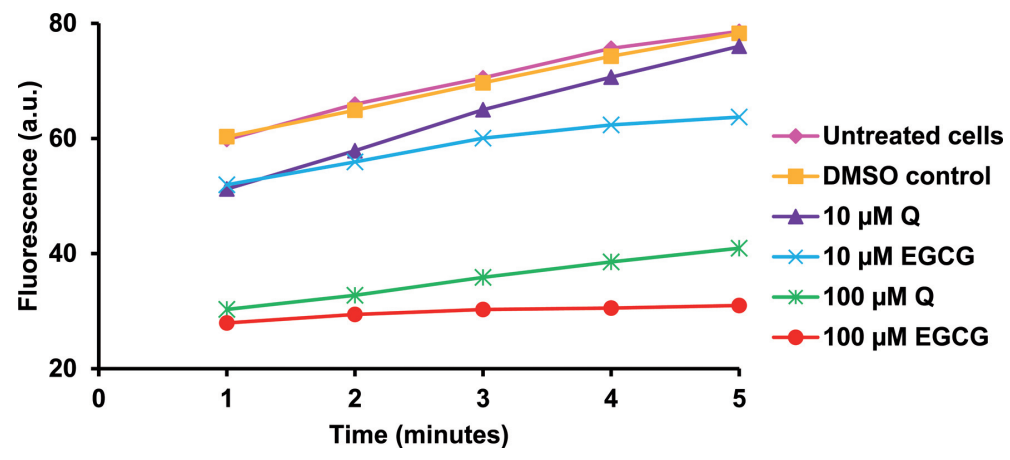

Figure 5. Effects of quercetin (Q) and EGCG on CuOOH-induced membrane peroxidation of Jurkat cells. DMSO has virtually no effect on the membrane susceptibility to lipid peroxidation. Q and EGCG inhibit the peroxidation with about $14 \%$ (at $\mathrm{t}_{1}$ ) for $10 \mu \mathrm{M}$ exposure level, and with about $50 \%$ (at $\mathrm{t}_{1}$ ) for $100 \mu \mathrm{M}$ concentrations. 
help to better understand their mechanism of action as antioxidants. Other prototypic free radical scavenging antioxidants have been shown to act as membrane stabilizers partly through a restriction of membrane fluidity. For example, it has been demonstrated that the antioxidant action of $a$-tocopherol is mediated through decreased membrane fluidity, which is equivalent to the increase of the membrane anisotropy (Kagan et al. 1990; Arora et al. 2000). On the other hand, some toxic effects of xenobiotics or other factor (such as viruses) have been connected to the increase of the membrane fluidity (Sergent et al. 2005, 2009; Mishra et al. 2009).

Results obtained in this study on constitutively proliferating cell lines - U937 monocytes and Jurkat T lymphoblasts were not in agreement with previous results obtained by us with freshly isolated mononuclear cells from peripheral blood. Margina et al. (2010) showed that peripheral blood mononuclear cells isolated from healthy subjects exhibit a decrease of the anisotropy value, when exposed for 20 minutes to quercetin $(1 \mu \mathrm{M})$ and EGCG $(10 \mu \mathrm{M})$. The different effects of quercetin and EGCG, depending on the cellular model might be explained considering that primary mononuclear cells are heterogeneous in size (monocytes and lymphocytes). Moreover, in our previous studies on mononuclear cells, we used lower concentrations of quercetin and thus it is difficult to compare results obtained on cell lines and freshly isolated human cells.

In the present study we wanted to prove that the antioxidant properties of the two tested polyphenols - quercetin and EGCG - are linked to their stabilizing effect on the cell membrane. In order to do so, we tested the outcome of the short-time exposure of two suspension cell lines (Jurkat and U937) to different concentrations of quercetin and EGCG, in the range $10 \mu \mathrm{M}$ to $100 \mu \mathrm{M}$. Data from the literature state that polyphenol plasma physiological level is about $2-10 \mu \mathrm{M}$, levels of $20-100 \mu \mathrm{M}$ being considered as supraphysiological concentrations (Winterbone et al. 2009). Therefore the concentration interval was chosen in order to cover both the physiological and supraphysiological levels. We assayed the results of the cell-flavonoid interaction in terms of membrane anisotropy, transmembrane potential as well as $\mathrm{CuOOH}$ induced lipid peroxidation.

Our results performed on two cell lines (Jurkat and U937) showed that quercetin and EGCG induced a concentration dependent increase of the membrane anisotropy, and therefore the decrease of the membrane fluidity (membrane stiffening).

We used $\mathrm{DiBAC}_{4}(3)$ to evaluate the effect of the tested compounds on the transmembrane potential; the decrease of the bound to cell dye fluorescence indicated a hyperpolarization effect of the polyphenols on the cell membranes. The effect is also dose-dependent for both U937 and Jurkat cells.
In order to evaluate the outcome of the polyphenol-cell membrane interaction in terms of redox status, we used DPPP as a fluorescent probe. According to literature date, hydroperoxides generated in cell membranes, lipoproteins, serum, tissues or other type of biological sample can be directly detected using the fluorogenic reagent DPPP which is essentially non-fluorescent until oxidized to a phosphineoxide by peroxides. Due to its solubility in lipids, DPPP intercalates into the membrane leaflets and reacts with lipid hydroperoxides, thus allowing the evaluation of peroxide formation in the membranes of live cells (Kawai et al. 2007). The lipid peroxidation of the membranes was induced with $\mathrm{CuOOH}$, which reacts more effectively with DPPP, compared to other chemical systems.

Under oxidative stress condition induced with $\mathrm{CuOOH}$, the tested polyphenols induced a dose dependent (10-100 $\mu \mathrm{M}$ ) decrease of DPPP fluorescence in the membranes of live cells. Since quercetin and EGCG have a membrane stabilizing effect that is similar to that documented by literature for $\alpha$-tocopherol, this mechanism may partly account for their antioxidant activities (van Aalst et al. 2004).

Results showed that, at concentrations higher than 50 $\mu \mathrm{M}$, for both tested compounds and for both cell lines, the effect on anisotropy and transmembrane potential reached saturation. Cells were checked for viability after 20 minutes incubation with quercetin and EGCG, using Trypan blue as vital dye. We found that effect saturation was not due to cell death. Lugli et al. (2009) state that 50 and $100 \mu \mathrm{M}$ of quercetin induced significant DNA degradation in U937 cells, only after significantly longer incubation time ( $24 \mathrm{~h})$ than that used in our model. Additionally, Ramos and Aller (2008) showed that apoptosis is induced by $25 \mu \mathrm{M}$ of quercetin after $8 \mathrm{~h}$ of incubation and increases at longer incubation times.

Taking into account the reduced effect proved using Trypan blue and the short time of incubation of the tested polyphenols with the cell lines, and also, considering literature data that report apoptosis induction after several hours of incubation with the polyphenols, we considered that cytotoxicity is not relevant for the overall conclusions, in the setting of our experiments.

Saturation might be related to a critical maximal number of polyphenol molecules which cell membranes can incorporate.

While for U937 cells both polyphenols (up to $100 \mu \mathrm{M}$ ) induced similar increase of membrane anisotropy, in the case of Jurkat cells the effects were significantly different. Quercetin induced an almost $60 \%$ gradual increase of $r$, when used in concentrations up to $50 \mu \mathrm{M}$, thereafter the effect being limited. EGCG induced a lower effect (maximum of $11.16 \%$ increase at $100 \mu \mathrm{M}$ exposure). This difference was probably due to the differences between the membrane structures of the two cell lines, which strongly 
influence their interaction with the tested components. We highlight that U937 cells are monocytic in origin and are functionally designed for increased endocytosis when compared to Jurkat lymphoblasts, and this might explain, at least partially, the behavior differences between the two cell lines.

Comparing the results obtained for the membrane anisotropy to that one concerning the antioxidant potential of the polyphenols, we can conclude that the increase of the membrane anisotropy is correlated with the dose-dependent increase of the antioxidant potential for the two tested compounds.

At polyphenol concentrations up to about $50 \mu \mathrm{M}$ a similar, gradual hyperpolarizing effect was induced by both tested compounds. At higher concentrations of quercetin, hyperpolarization increased dramatically $(17.14 \%)$, while increasing the concentration of EGCG has resulted in only a small increase of transmembrane potential (3.67\%).

Concentration profiles of hyperpolarization of Jurkat cells for both polyphenols are similar to those observed in U937 cells, with the difference that the level of hyperpolarization is markedly different at low concentrations of polyphenols; the EGCG is significantly more efficient.

A significant increase of hyperpolarization, particularly obvious for quercetin, occurred at concentrations above $50 \mu \mathrm{M}$, when the anisotropy reached a maximum level (with the exception of $200 \mu$ M EGCG in U937 cells, Fig. 1 - a single point). This fact indicated that the maximum number of polyphenol molecules in cell membranes was achieved, which is sufficient to significantly narrow pore formation and outflow of small inorganic ions (like $\mathrm{K}^{+}$) from the cells, resulting in a significant membrane hyperpolarization.

By inducing a greater degree of membrane "stiffening" and hyperpolarization, the tested compounds could reduce the mobility of free radicals in the lipid bilayer. The decreased membrane fluidity could lead to the inhibition of lipid peroxidation.

Acknowledgements. The work was performed under the CNCSIS grant PD 132/30.07.2010 (PD 29/2010).

\section{References}

Arora A., Byrem T. M., Nair M. G., Strasburg G. M. (2000): Modulation of liposomal membrane fluidity by flavonoids and isoflavonoids. Arch. Biochem. Biophys. 373, 102-109 http://dx.doi.org/10.1006/abbi.1999.1525

Arts I. C., Jacobs D. R. Jr., Gross M., Harnack L. J., Folsom A. R. (2002): Dietary catechins and cancer incidence among postmenopausal women: the Iowa Women's Health Study (United States). Cancer Causes Control 13, 373-382 http://dx.doi.org/10.1023/A:1015290131096
Barros M. P., Pinto E., Colepicolo P., Pedersen M. (2001): Astaxanthin and peridinin inhibit oxidative damage in $\mathrm{Fe}(2+)$ loaded liposomes: scavenging oxyradicals or changing membrane permeability? Biochem. Biophys. Res. Commun. 288, 225-232 http://dx.doi.org/10.1006/bbrc.2001.5765

Boots A. W., Haenen G., Bast A. (2008): Health effects of quercetin: From antioxidant to nutraceutical. Eur. J. Pharmacol. $\mathbf{5 8 5}, 325-337$ http://dx.doi.org/10.1016/j.ejphar.2008.03.008

Breusing N., Grune T., Andrisic L., Atalay M., Bartosz G., Biasi F., Borovic S., Bravo L., Calals I., Casillas R. et al. (2010): An inter-laboratory validation of methods of lipid peroxidation measurement in UVA-treated human plasma samples. Free Radic. Res. 44, 1203-1215 http://dx.doi.org/10.3109/10715762.2010.499907

Epps D. E., Wolfe M. L., Groppi V. (1994): Characterization of the steady-state and dynamic fluorescence properties of the potential-sensitive dye bis-(1,3-dibutylbarbituric acid)trimethine oxonol (Dibac4(3)) in model systems and cells. Chem. Phys. Lipids 69, 137-150 http://dx.doi.org/10.1016/0009-3084(94)90035-3

Erlejman A. G., Jaggers G., Fraga C. G., Oteiza P. I. (2008): TNFalpha-induced NF-kappaB activation and cell oxidant production are modulated by hexameric procyanidins in Caco-2 cells. Arch. Biochem. Biophys. 476, 186-195 http://dx.doi.org/10.1016/j.abb.2008.01.024

Fraga C. G., Galleano M., Verstraeten S. V., Oteiza P. I. (2010): Basic biochemical mechanisms behind the health benefits of polyphenols. Mol. Aspects Med. 31, 435-445 http://dx.doi.org/10.1016/j.mam.2010.09.006

Fujimoto K., Iwasaki C., Kawaguchi H., Yasugi E., Oshima M. (1999): Cell membrane dynamics and the induction of apoptosis by lipid compounds. FEBS Lett. 446, 113-116 http://dx.doi.org/10.1016/S0014-5793(99)00204-5

Ghosh P. K., Vasanji A., Murugesan G., Eppell S. J., Graham L. M., Fox P. L. (2002): Membrane microviscosity regulates endothelial cell motility. Nat. Cell. Biol. 4, 894-900 http://dx.doi.org/10.1038/ncb873

Gomes A., Fernandes E., Lima J. L. (2005): Fluorescence probes used for detection of reactive oxygen species. J. Biochem. Biophys. Methods 65, 45-80 http://dx.doi.org/10.1016/j.jbbm.2005.10.003

Huang Z. J., Haugland R. P. (1991): Partition coefficients of fluorescent probes with phospholipid membranes. Biochem. Biophys. Res. Commun. 181, 166-171 http://dx.doi.org/10.1016/S0006-291X(05)81396-8

Iftime A., Popescu A., Ganea C. (2010): Effects of epigallocatechin gallate on artificial lipid membranes. Romanian J. Biophys. 20, 323-334

Ilie M., Margina D., Katona E., Ganea C., Pencea C., Gradinaru D., Vladica M., Mitrea N., Balalau D. (2009): Quercetin and epigallocatechin gallate effect on the lipid order parameter of peripheral blood mononuclear cells from diabetes patients. Rom. Biotechnol. Lett. 14, 4804-4811

Kagan V. E., Serbinova E. A., Bakalova R. A., Stoytchev T. S., Erin A. N., Prilipko L. L., Evstigneeva R. P. (1990): Mechanisms of stabilization of biomembranes by alpha-tocopherol: The role of 
the hydrocarbon chain in the inhibition of lipid peroxidation. Biochem. Pharmacol. 40, 2403-2413 http://dx.doi.org/10.1016/0006-2952(90)90080-5

Kajiya K., Kumazawa S., Nakayama T. (2002): Effects of external factors on the interaction of tea catechins with lipid bilayer. Biosci. Biotechnol. Biochem. 66, 2330-2335 http://dx.doi.org/10.1271/bbb.66.2330

Kamihira M., Nakazawa H., Kira A., Mizutani Y., Nakamura M., Nakayama T. (2008): Interaction of tea catechins with lipid bilayers investigated by a quartz-crystal microbalance analysis. Biosci. Biotechnol. Biochem. 72, 1372-1375 http://dx.doi.org/10.1271/bbb.70786

Kanupriya L., Dipti P., Sharma S. K., Sairam M., Ilavazhagan G., Sawhney R. C., Barnejee P. K. (2006): Flavonoids protect U-937 macrophages against tert-butylhydroperoxide induced oxidative injury. Food Chem. Toxicol. 44, 1024-1030 http://dx.doi.org/10.1016/j.fct.2005.12.005

Kawai Y., Miyoshi M., Moon J. H., Terao J. (2007): DPPP-TLC blot/GC-MS for lipid hydroperoxide isomers. Anal. Biochem. 360, 130-137 http://dx.doi.org/10.1016/j.ab.2006.09.028

Klapperstück T., Glanz D., Klapperstück M., Wohlrab J. (2009): Methodological aspects of measuring absolute values of membrane potential in human cells by flow cytometry. Cytometry A 75, 593-608

Kumazawa S., Kajiya K, Naito A., Saito H., Tuzi S., Tanio M., Suzuki M., Nanjo F., Suzuki E., Nakayama T. (2004): Direct evidence of interaction of a green tea polyphenol, epigallocatechin gallate, with lipid bilayers by solid-state nuclear magnetic resonance. Biosci. Biotechnol. Biochem. 68, 1743-1747 http://dx.doi.org/10.1271/bbb.68.1743

Lakowicz J. R. (1999): Principles of Fluorescence Spectroscopy (2nd edition). Kluwer Academic/Plenum, New York

Lugli E., Ferraresi R., Roat E., Troiano L., Pinti M., Nasi M., Nemes E., Bertoncelli L. et al. (2009): Quercetin inhibits lymphocyte activation and proliferation without inducing apoptosis in peripheral mononuclear cells. Leuk. Res. 33, 140-150 http://dx.doi.org/10.1016/j.leukres.2008.07.025

Manach C., Scalbert A., Morand C., Remesy C., Jimenez L. (2004) Polyphenols: food sources and bioavailability. Am. J. Clin. Nutr. 79, 727-747

Margina D., Ilie M., Negrei C., Gradinaru D., Balanescu A., Mitrea N. (2010): Quercetin and epigallocatechin gallate in vitro induced changes in the membrane anisotropy of peripheral blood mononuclear cells from patients with inflammatory diseases. J. Med. Plants Res. 22, 2388-2392

Margina D., Gradinaru D., Panaite C., Cimponeriu D., Vladica M., Danciulescu R., Mitrea N. (2011): The association of adipose tissue markers for redox imbalance and the cardio-vascular risk in obese patients. HealthMED 5, 194-199

Martins A. P., Lopes P. A., Martins S. V., Madeira A., Santos N. C., Prates J. A. M., Moura T. F., Soveral G. (2010): Conjugated linoleic acid reduces permeability and fluidity of adipose plasma membranes from obese Zucker rats. Biochem. Biophys. Res. Commun. 398, 199-204

http://dx.doi.org/10.1016/j.bbrc.2010.06.059

Mishra M. K., Ghosh D., Duseja R., Basu A. (2009): Antioxidant potential of Minocycline in Japanese Encephalitis Virus infection in murine neuroblastoma cells: Correlation with membrane fluidity and cell death. Neurochem. Int. 54, 464-470 http://dx.doi.org/10.1016/j.neuint.2009.01.022

Mursu J., Voutilainen S., Nurmi T., Tuomainen T. P., Kurl S., Salonen J. T. (2008): Flavonoid intake and the risk of ischaemic stroke and CVD mortality in middle-aged Finnish men: the Kuopio ischaemic heart disease risk factor. Study Br. J. Nutr. 100, 890-895

Okimoto Y., Watanabe A., Niki E., Yamashita T., Noguchi N. (2000): A novel fluorescent probe diphenyl-1-pyrenylphosphine to follow lipid peroxidation in cell membranes. FEBS Lett. 474, $137-140$ http://dx.doi.org/10.1016/S0014-5793(00)01587-8

Oteiza P. I., Erlejman A. G., Verstraeten S. V., Keen C. L., Fraga C. G. (2005): Flavonoid-membrane interactions: a protective role of flavonoids at the membrane surface? Clin. Dev. Immunol. 12, 19-25 http://dx.doi.org/10.1080/10446670410001722168

Pebay-Peyroula E., Dufourc E. J., Szabo A. G. (1994): Location of diphenyl-hexatriene and trimethylammonium-diphenylhexatriene in dipalmitoylphosphatidylcholine bilayers by neutron diffraction. Biophys. Chem. 53, 45-56 http://dx.doi.org/10.1016/0301-4622(94)00075-1

Ramos A. M., Aller P. (2008): Quercetin decreases intracellular GSH content and potentiates the apoptotic action of the antileukemic drug arsenic trioxide in human leukemia cell lines. Biochem. Pharmacol. 75, 1912-1923 http://dx.doi.org/10.1016/j.bcp.2008.02.007

Sánchez-Gallego J. I., López-Revuelta A., Sardina J. L., Hernández-Hernández A., Sánchez-Yagüe J., Llanillo M. (2010): Membrane cholesterol contents modify the protective effects of quercetin and rutin on integrity and cellular viability in oxidized erythrocytes. Free Radic. Biol. Med. 48, $1444-1454$

http://dx.doi.org/10.1016/j.freeradbiomed.2010.02.034

Schroeter H., Heiss C., Balzer J., Kleinbongard P., Keen C. L., Hollenberg N. K., Sies H., Kwik-Uribe C., Schmitz H. H., Kelm M. (2006): (-)-Epicatechin mediates beneficial effects of flavanolrich cocoa on vascular function in humans. Proc. Natl. Acad. Sci. U.S.A. 103, 1024-1029

http://dx.doi.org/10.1073/pnas.0510168103

Sergent O., Pereira M., Belhomme C., Chevanne M., Huc L., Lagadic-Gossmann D. (2005): Role for membrane fluidity in ethanol-induced oxidative stress of primary rat hepatocytes. J. Pharmacol. Exp. Ther. 313, 104-111 http://dx.doi.org/10.1124/jpet.104.078634

Sergent O., Ekroos K., Lefeuvre-Orfila L., Rissel M., Forsberg G. B., Oscarsson J., Andersson T.B., Lagadic-Gossmann D. (2009): Ximelagatran increases membrane fluidity and changes membrane lipid composition in primary human hepatocytes. Toxicol. in Vitro 23, 1305-1310 http://dx.doi.org/10.1016/j.tiv.2009.07.019

Takahashi M., Shibata M., Niki E. (2001): Estimation of lipid peroxidation of live cells using a fluorescent probe, diphenyl1-pyrenylphosphine. Free Radic. Biol. Med. 31, 164-174 http://dx.doi.org/10.1016/S0891-5849(01)00575-5

Tamba Y., Ohba S., Kubota M., Yoshioka H., Yoshioka H., Yamazaki M. (2007): Single GUV method reveals interaction of tea cat- 
echin - epigallocatechin gallate with lipid membranes. Biophys. J. 92, 3178-3194 http://dx.doi.org/10.1529/biophysj.106.097105

Taubert D., Berkels R., Roesen R., Klaus W. (2003): Chocolate and blood pressure in elderly individuals with isolated systolic hypertension. J. Am. Med. Assoc. 290, 1029-1030 http://dx.doi.org/10.1001/jama.290.8.1029

van Aalst J. A., Burmeister W., Fox P. L., Graham L. M. (2004) Alpha-tocopherol preserves endothelial cell migration in the presence of cell-oxidized low-density lipoprotein by inhibiting changes in cell membrane fluidity. J. Vasc. Surg. 39, 229-237 http://dx.doi.org/10.1016/S0741-5214(03)01038-3

Van Blitterswijk W. J., Van Hoeven R. P., Van Der Meer B. W. (1981): Lipid structural order parameters (reciprocal of fluidity) in biomembranes derived from steady state fluorescence polarization measurements. Biochim. Biophys. Acta 644, 323-332 http://dx.doi.org/10.1016/0005-2736(81)90390-4
Verstraeten S. V., Mackenzie G. G., Oteiza P. I., Fraga C. G. (2008): (-)-Epicatechin and related procyanidins modulate intracellular calcium and prevent oxidation in Jurkat $\mathrm{T}$ cells. Free Radic. Res. 42, 864-872

http://dx.doi.org/10.1080/10715760802471452

Vita J. A. (2005): Polyphenols and cardiovascular disease: effects on endothelial and platelet function. Am. J. Clin. Nutr. 81, S292-297

Winterbone M. S., Tribolo S., Needs P. W., Kroon P. A., Hughes D. A. (2009): Physiologically relevant metabolites of quercetin have no effect on adhesion molecule or chemokine expression in human vascular smooth muscle cells. Atherosclerosis 202, 431-438 http://dx.doi.org/10.1016/j.atherosclerosis.2008.04.040

Received: September 19, 2011

Final version accepted: November 22, 2011 